\title{
EXPERIMENTAL INVESTIGATION OF THE AERODYNAMIC AND THERMAL BEHAVIOUR OF THE FILM AT THE LEADING EDGE OF A COOLED NOZZLE VANE CASCADE
}

\section{Luca Casarsa ${ }^{1}$ Fabio Pagnacco ${ }^{1}$, Hamed Abdeh ${ }^{2}$, Giovanna Barigozzi ${ }^{2}$}

${ }^{1}$ Dipartimento Politecnico di Ingegneria e Architettura - Università di Udine - Udine, Italy luca.casarsa@uniud.it, fabio.pagnacco@uniud.it

${ }^{2}$ Dipartimento di Ingegneria e Scienze Applicate - Università di Bergamo - Dalmine (BG) Italy hamed.abdeh@nibg.it, giovanna.barigozzi@unibg.it

\begin{abstract}
This paper presents the results of an experimental investigation of the aerodynamic behavior of coolant ejection at the leading edge of a nozzle vane cascade. Highly spatially resolved Particle Image Velocimetry (PIV) data were acquired on mid span blade-to-blade planes and along the span wise direction to capture the 3D mixing process. Complementary Pressure Sensitive Paints (PSP) data are used to support the aerodynamic analysis. Tests were performed at a fixed cascade operating condition $\left(\mathrm{Ma}_{2 \mathrm{is}}=0.2\right.$ and $\left.\mathrm{Tu} \mathrm{u}_{1}=\mathbf{1 . 6 \%}\right)$ varying the coolant to main stream blowing ratio (BR) from 2.0 up to 4.0. Flow filed data clearly show a shift in stagnation and a strong jet to jet as well as jet to mainstream interaction for all tested $B R$, supporting the unsteady and highly anisotropic nature of the phenomenon.
\end{abstract}

KEYWORDS: Gas Turbine, Film Cooling, Showerhead, PIV

$\begin{array}{ll}\text { NOMENCLATURE } & \\ A & \text { cross section } \\ B R & \text { blowing ratio } \\ C & \text { chord } \\ H & \text { vane height } \\ m & \text { mass flow } \\ M a & \text { Mach number } \\ N & \text { direction normal to the wall } \\ S & \text { surface curvilinear coordinate } \\ S & \text { pitch } \\ T u & \text { in plane turbulence intensity level } \\ U_{l} & \text { inlet free stream velocity } \\ \text { Vel } & \text { in plane mean velocity } \\ \text { Vort } & \text { vorticity } \\ X, Y, Z & \text { cascade coordinate system } \\ \eta & \text { film cooling effectiveness } \\ \rho & \text { flow density } \\ \text { Subscript } & \\ c & \text { coolant } \\ l & \text { inlet freestream condition }\end{array}$




\section{INTRODUCTION}

As well known, in modern gas turbines the leading edge region of nozzle vane and rotor blade cascades need a highly efficient cooling system to protect these components from the high temperature gas coming from the combustor. Complex internal cooling systems are commonly adopted in combination with a dense array of cylindrical rather than shaped film cooling holes (Bogard and Thole, 2006). The design of these cooling holes, typically referred as showerhead, strongly depends on the approaching flow condition as well as on the leading edge shape (Chavez et al., 2007, Jenkins et al. ,2004). In fact, both hot streak and inlet flow swirl have an impact on the thermal performance of each specific cooling scheme, since they influence the heat load to the vane and the stagnation line position. The thermal performance of showerhead cooling systems is well documented in the open literature in terms of film cooling effectiveness and, less often, heat transfer coefficient. In this context the impact of operating conditions such as blowing and density ratio and mainstream turbulence has been clarified on specific hole geometries (see for example Mhetras et al. ,2012, Li et al. 2014). These studies demonstrated that in most showerhead cooling schemes the operating conditions typically result in significant jet liftoff phenomena, particularly relevant in the stagnation region. Although an abundant literature does exist on the thermal performance of these cooling systems, their aerodynamic performance is not deeply analyzed. Only a few papers document the strong mixing between coolant jets and the mainstream, showing the appearance of huge and highly anisotropic turbulence levels and a modification in the stagnation line position induced by coolant injection (Cutbirth and Bogard, 2001 and Polanka et al. ,2002). In these papers the influence of mainstream turbulence intensity level at a fixed blowing ratio $(B R=2)$ is investigated by means of flow visualizations and the 3D Laser Doppler Anemometry technique, the latter used only in the mid span plane.

In the last few years, the progress in Computational Fluid Dynamics (CFD) allows for the simulation of complex geometries and operating conditions. The typical experimental approach has been thus complemented with numerical simulations, allowing to test the prediction capabilities of conventional (Dyson et al., 2012) and advanced (Rozati and Tafti, 2008, and Ravelli and Barigozzi, 2017) approaches in solving the complex phenomena related to the jet to mainstream mixing process in the stagnation region. This specific test condition is particularly challenging for the computational approach, because of the presence of detached jets and of jet to jet as well as jet to mainstream interactions, the whole taking place in a region of strong acceleration. The availability of detailed and quantitative information on the unsteady $3 \mathrm{D}$ flow structures developing around the stagnation region of a showerhead cooling system is of value for the comprehension of the complex flow phenomena taking place in that region and they will support the thermal characterizations that are more often performed on these cooling schemes. Last but not least they are essential for the validation of CFD simulations, especially when hybrid RANS/LES approaches are considered.

In this context this paper presents the results of an experimental investigation performed as a collaboration between Bergamo and Udine Universities whose aim was to provide detailed information on the unsteady flow behavior in the leading edge region of a showerhead cooled nozzle vane cascade. Highly spatially resolved PIV measurements were performed using air as coolant with variable injection conditions corresponding to $B R=2.0,3.0$ and 4.0. Data belonging to the $B R=3.0$ case have been the object of a specific paper (Barigozzi et al., 2019)). Some of these data are used here as a reference. Differently from past investigations, in this paper data are not limited to the mid span region, but information on the flow evolution along the vane span is also provided. The same cascade geometry and cooling scheme have been also investigated in the past in terms of film cooling effectiveness distributions for variable injection conditions (Barigozzi and Ravelli, 2015). Thermal data extracted from selected locations are used here to have a complete overview of the aero-thermal performance of the tested geometry. 


\section{TEST RIG, VANE CONFIGURATION, AND EXPERIMENTAL METHODOLOGY}

\section{Test rig and vane geometry}

Tests were performed in the continuously operating, suction-type subsonic wind tunnel for nozzle vane cascade (Fig. 1a) installed at the Energy Systems and Turbomachinery Laboratory of Bergamo University and in cooperation with Udine University research team. The wind tunnel configuration is such to guarantee the optical accessibility to perform either heat transfer or flow field measurements by means of optical methods. The nozzle model consists in a high pressure 6 vane cascade typical of an industrial gas turbine. The central vane presents a showerhead cooling system, whose details can be appreciated in Fig. 1b. All the details about the wind tunnel and the cascade geometry can be found in Barigozzi and Ravelli (2015) and for this reason only the most significant information is provided here. The cascade, manufactured in polished Plexiglas, is characterized by a pitch to chord ratio of 1.0 and an aspect ratio of 0.69 . The vane chord $C$ and height $H$ are $142.1 \mathrm{~mm}$ and $98 \mathrm{~mm}$, respectively. The showerhead cooling scheme features four staggered rows of 16 cylindrical holes (of diameter $D=1 \mathrm{~mm}$, pitch equal to $5.88 D$ along the span and row spacing equal to $5.1 D$ ) covering $90 \%$ of the vane span. Holes are injecting towards the vane outer hub with an injection angle of $45^{\circ}$; the injection angle in the stream wise direction is equal to $90^{\circ}$. Cooling holes are fed by a single plenum realized inside the vane (Fig. 1b) and connected at the inner hub side to the compressed air delivering system of the facility.

a)

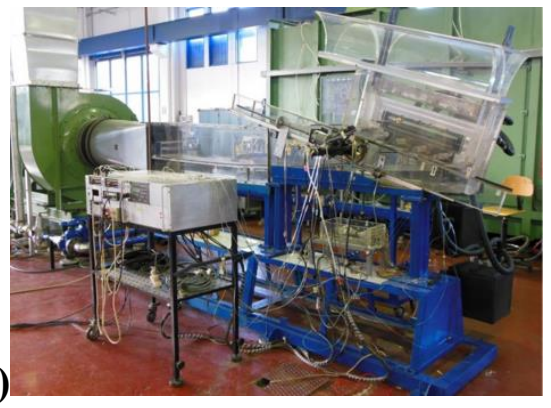

b)

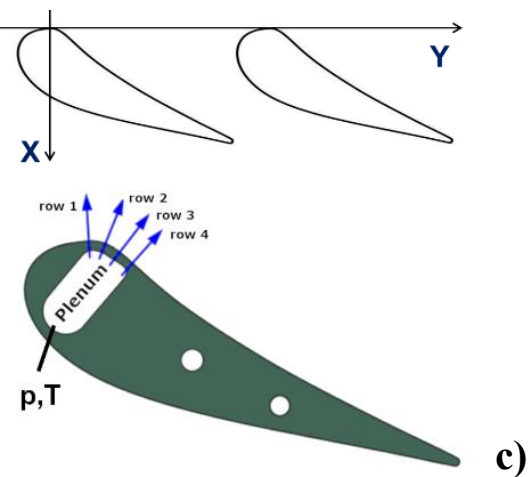

c)

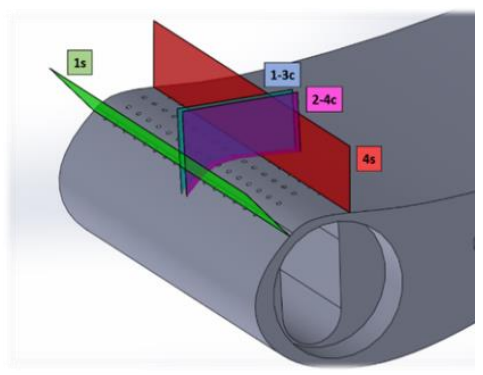

Figure 1: The wind tunnel (a), the cascade model (b) and the measurement planes (c).

\section{Test conditions}

A low turbulence $\left(T u_{1}=1.6 \%\right)$ and low speed testing condition was adopted in these experiments, corresponding to an inlet Mach number $M a_{1}$ of 0.063 and to an exit isentropic Mach number $M a_{2 i s}$ of 0.2. This testing condition is below the design inlet Mach number for this vane which is set at 0.12 . This reduction was necessary in order to provide an acceptable operating condition for the PIV technique, and it was considered acceptable since the focus of this investigation was on the leading edge region. Please note that such a Mach number reduction does not translate into a modification of the stagnation position. This was verified measuring the vane load distribution for variable $M a_{2 i s}$ in the range $0.2-0.6$. The cooling line was fed with compressed air at three different flow rates to match coolant to mainstream blowing ratio $B R$ values of 2,3 and $4 . B R$ is defined as follows:

$$
B R=\frac{\rho_{c} U_{c}}{\rho_{1} U_{1}}=\frac{m_{c}}{m_{1}} \cdot \frac{A_{1}}{A_{c}}
$$

$A_{c}$ and $A_{l}$ are the coolant hole exit section area and the mainstream cascade inlet section area, respectively. The density ratio was about 1.0, due to the need to feed the cooling line with the seeding required to perform PIV tests.

\section{Particle Image Velocimetry set-up}

Flow filed measurements were performed by means of a Two-dimensional Particle Image Velocimetry (PIV) technique along four mutually perpendicular planes in an attempt to gain an almost 
3D view of the investigated flow field. All the details about instrumentation, data processing, determination of flow statistics, and uncertainty estimation are available in Barigozzi et al. (2019) and therefore are not reported for reason of shortness. Therefore, only the essential details that are necessary to understand the present results are provided in the following. Measurement planes were arranged as sketched in Fig. 1c. Planes 1-3c and 2-4c are blade-to-blade planes aligned with the 7th holes of rows \#1 and \#3 and the 7th holes of rows \#2 and \#4, respectively. Spanwise planes have been also considered, oriented normal to the vane surface and placed in correspondence of rows \#1 and \#4 (planes 1s and 4s of Fig. 1c). Problems associated to laser light reflection form the vane surface were minimized by adopting transparent blades with polished surface and background subtraction form the raw PIV images. By doing so, laser light back scatter was limited to about $0.2 \mathrm{~mm}$ of the vane surface.

In the following, flow field results will be presented only in terms of time averaged quantities or higher order statistics, such as turbulence intensity levels. They are the results of the ensemble averaging of 1000 uncorrelated instantaneous samples. In this case, the sampling error can be considered as an upper bound estimation of their uncertainty (because of the limited number of samples and of the random nature associated to the PIV evaluation algorithm used to obtain the instantaneous flow fields). Based on a $95 \%$ confidence level, the uncertainty in the mean velocity turns out to be less than $\pm 3 \%$ in most part of the flow fields, except for limited regions of very low velocity/high fluctuations at the boundaries of coolant jets. The uncertainty in the rms velocity fluctuations is always below $\pm 5 \%$.

\section{RESULTS AND DISCUSSION}

\section{Flow field in the blade-to-blade planes}

Figure 2 reports the contour plots of the time averaged in plane velocity $V_{e l}$ normalized with the approaching free stream velocity $U_{l}$ together with the stream tracers for the flow field measured in planes $1-3 \mathrm{c}$ and $2-4 \mathrm{c}$ for all the $B R$ conditions here considered. As already mentioned, data at $B R=3$ have been already presented in a previous contribution of the same authors but are here included to ease the comparison among different blowing conditions.
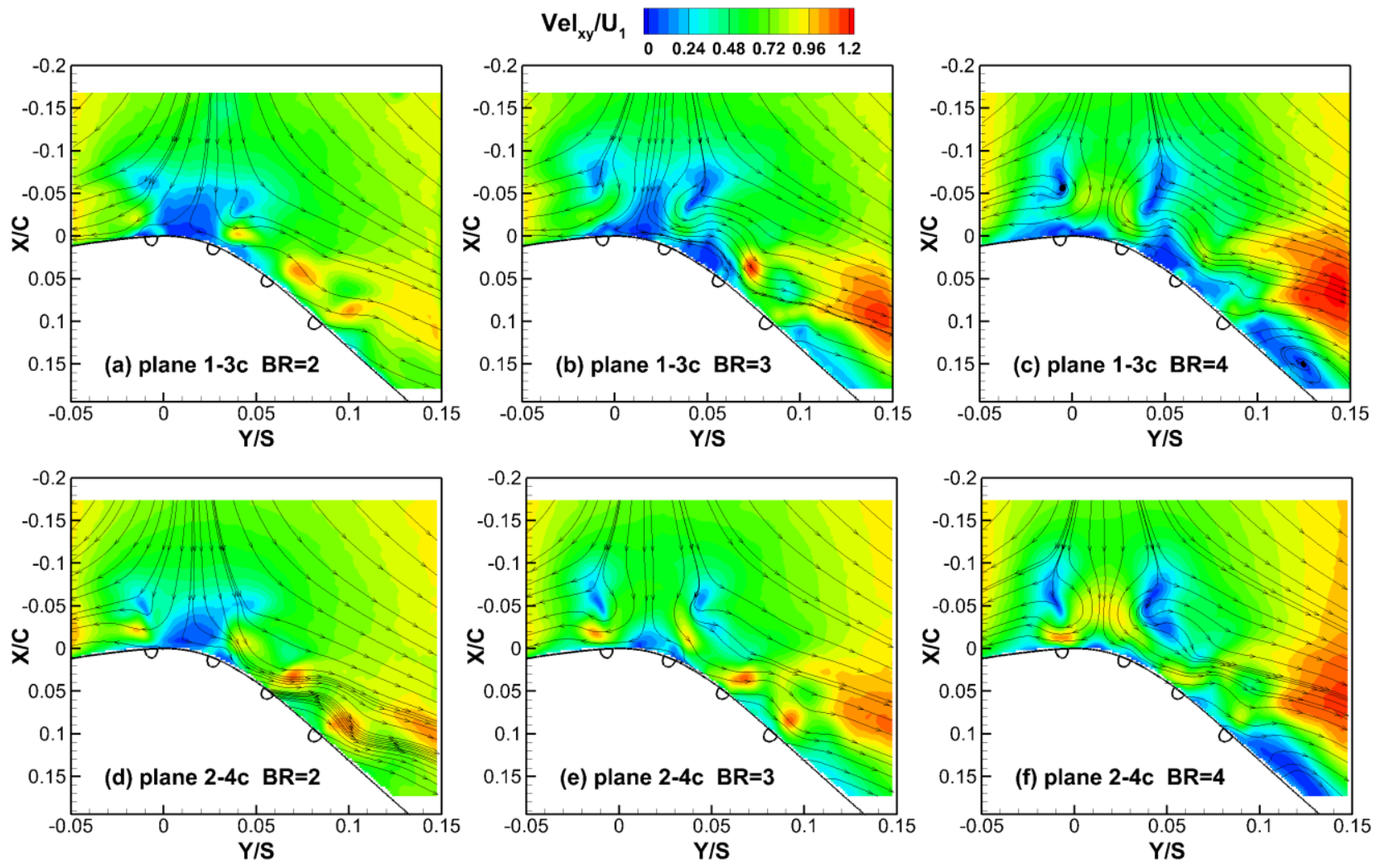
Figure 2: In plane normalized velocity distributions for variable $B R$ on the two blade to blade planes 1-3c (a-c) and 2-4c (d-e).

At a first glance, data show no significant differences in the mean flow behavior in the two flow planes at the same $B R$ and, similarly the same trends can be observed at increasing $B R$. The interaction between the coolant jets and the main flow is put in evidence by the stream tracers path and the local velocity distribution close to the vane surface. For $B R=2$ (Fig. 2a,d), the main flow appears to be significantly perturbed by the coolant mostly on the vane pressure side where local high velocity spots are found in correspondence of holes \#3 and \#4. A region of very low velocity is detected in between row\#1 and \#2 which pertains, in agreement with the stream tracers path, to the position of the stagnation point. This stagnation position does not change increasing $B R$ (Fig. 2c,f). This position of the stagnation line is not consistent with the vane design where a stagnation point downstream of row\#2 was detected by means of an instrumented vane. Therefore, jet to main stream interaction is confirmed to affect the location of stagnation line also for the present vane geometry and $B R$ conditions (Bohn et al., 1997 and Polanka et al., 2002). For $B R$ higher than 2, two regions of low in plane velocity are observed on both sides of stagnation. They are the footprint of jets coming from row\#1 and row\#2 injected at a lower elevation along the vane span with respect to the measurement plane. In particular, for $B R=4$, a local flow acceleration is observed in between these two jets, differently for the lower $B R$ cases where a flow deceleration can be appreciated. For $B R=4$, flow separation occurs downstream of row \#4, as also well evidenced by the very low flow agitation levels that can be observed in the contour plots of the in plane turbulence intensity of Fig. 3c. In this figure, only data pertaining to plane $2-4 \mathrm{c}$ are reported (same trends and distributions are found in plane $1-3 \mathrm{c}$ which are not reported for reason of space). The off the wall extension of the high fluctuations regions surrounding the vane leading edge provides an insight about jets penetration into the main flow. This region extends up to about $0.05 C$ for the case at $B R=2$ and widens up to about $0.15 C$ for the highest $B R$. Similarly, an increase of $B R$ determines higher fluctuations, also about the stagnation point, and the appearance of high fluctuations spots far from the wall. They are associated to jet lift-off and a more complex interaction with the main stream.
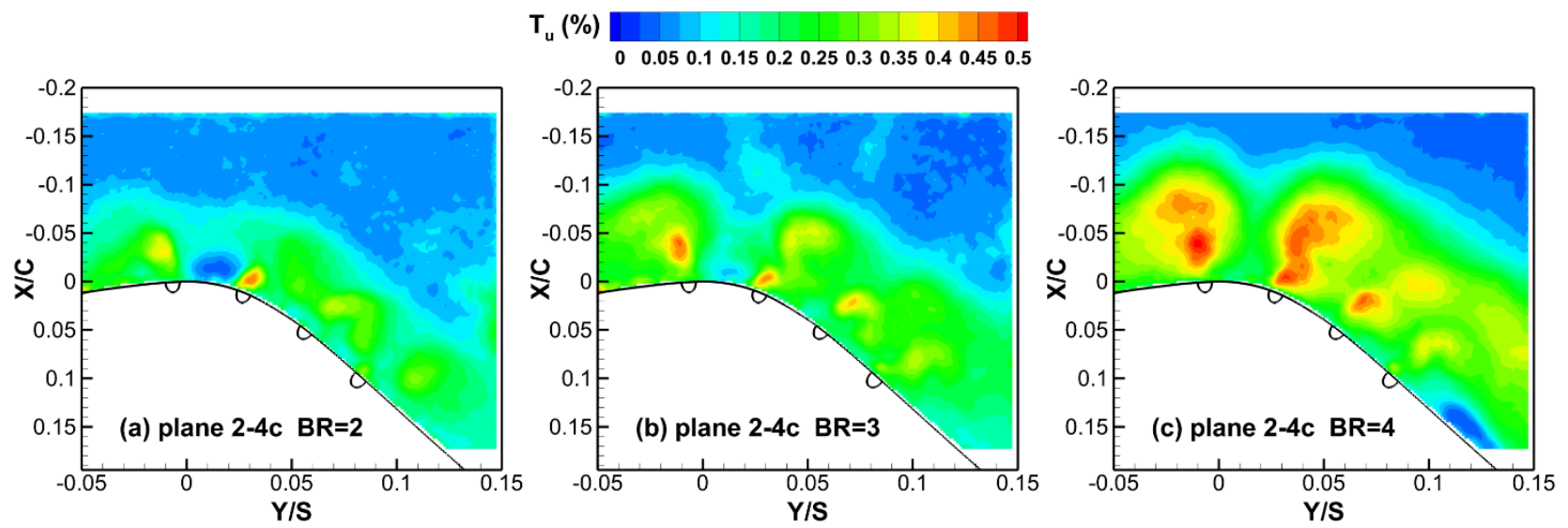

Figure 3: Turbulence intensity level for variable $B R$ on the blade to blade planes 2-4c (a-c).

To better clarify the turbulent behaviour of the leading edge flow, instantaneous flow fields were selected. Figure 4 reports examples of instantaneous velocity vectors, superimposed to the colour map of the in plane vorticity component, for the limit cases of $B R=2$ and 4 and obtained in plane 2-4c. These data have been selected (after the visual inspection of the whole population of instantaneous flow fields) as the best representative of the most likely conditions that occur. The first two frames belong to the $B R=2$ case while frames from (c) to (f) were collected at the largest $B R$ of 4 .

For the lower $B R$ (Fig. 4a-b), the coolant flow tends to remain well confined in a thin layer that surrounds the vane. Nevertheless, the main flow can penetrate this layer, as seen by the velocity 
vectors in between row \#1 and \#2 and downstream of row \#4. On the suction side, the instantaneous flow tends to move away from the wall, a further indication of the lift-off of jets from the first row. Very low velocity and vorticity values are found in between row $\# 1$ and $\# 2$. In the stagnation region a poor thermal protection is thus expected. For $B R=4$ the instantaneous flow is more complex. Figure 4-c reports an instantaneous flow condition where jets 1 and 2 strongly penetrate the main flow and cause the generation of two wide and intense recirculating structures that bound the incoming flow to the stagnation. The main flow passes under the jets and sweeps the vane surface. These vortices are not stable in time, as can be seen in Fig. 4d-f where the footprints of the first two jets are easily detected far away from the surface. In Fig. 4c-f the existence of the separated flow region downstream of row $\# 4$ can be appreciated by the velocity and vorticity distribution. In general, the vorticity field at $B R=4$ appears more scattered than at $B R=2$, confirming the stronger and more complex mixing process that takes place between coolant and main stream, as already observed when commenting the turbulence data.
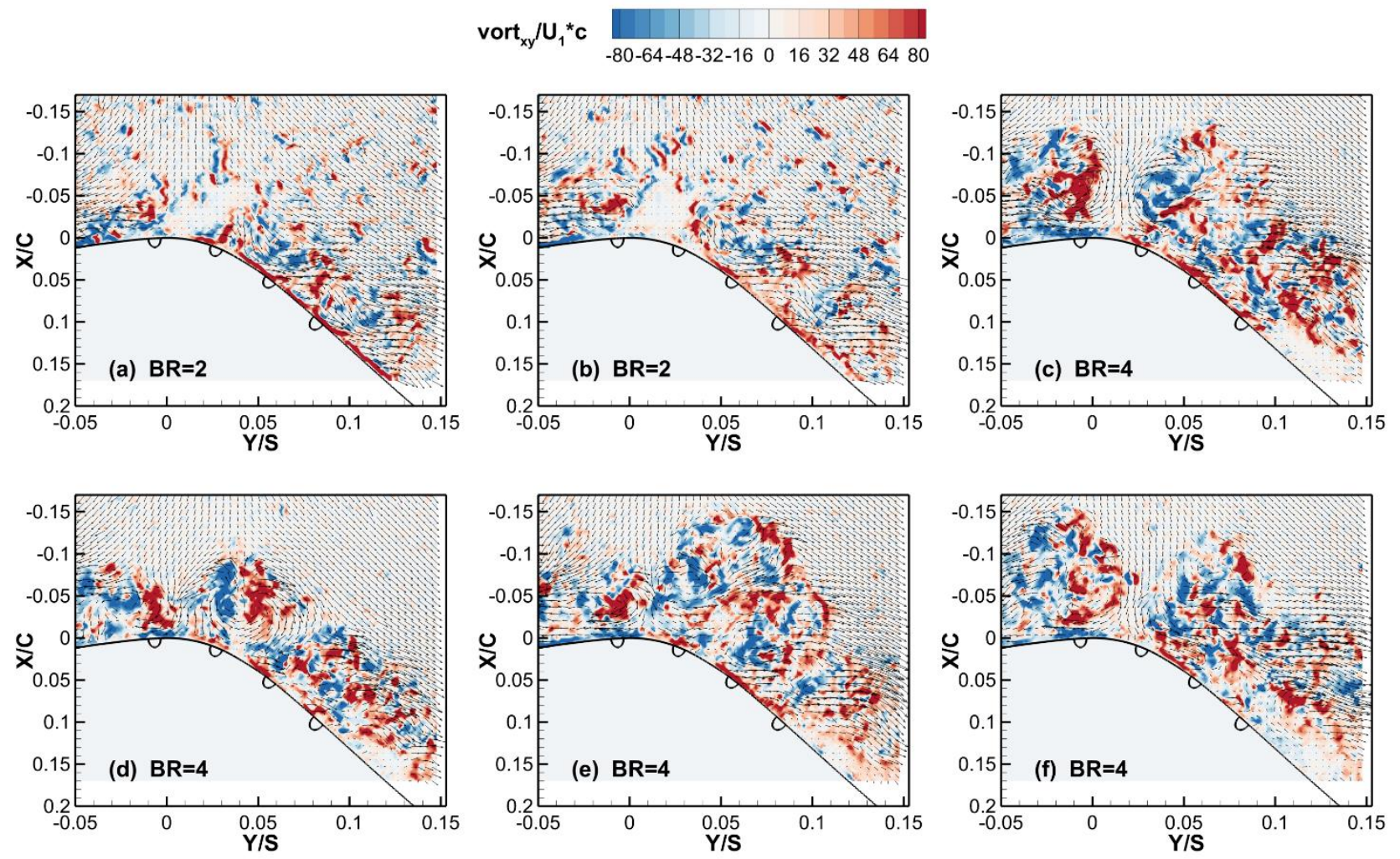

Figure 4: Instantaneous velocity vectors superimposed to vorticity maps for $B R=2.0$ (a-b) and $B R=4.0$ (c-f) on the blade to blade planes 2-4c.

The limitation in the temporal resolution of the available PIV system did not allow to track in time the evolution of the instantaneous flow field, nor to make a spectral analysis to look for possible dominant frequencies. Nevertheless, a proper orthogonal decomposition (POD) analysis was performed on the full population of instantaneous velocity fields, which results did not show any dominant mode in the flow. This confirms the random nature of the mixing phenomena previously commented.

The aerodynamic behaviour of the flow on the blade to blade planes just introduced is consistent and helps to understand the thermal performance measured by means of the PSP technique for the same operating conditions. To complement the aerodynamic analysis with the thermal point of view, local values of film cooling effectiveness data are here reported and summarized in Fig. 5. Data have been extracted at the same location where the PIV data have been acquired (i.e. locations of planes $1-3 c$ and 2-4c). For all conditions and at both locations, a general poor protection is observed downstream of each row of jets, consistently with jets separation and mixing with the main stream. 
Only jets from row\#1 contribute to the cooling of the suction side, while coolant accumulation from row\#2, \#3, and \#4 contributes to an increased thermal protection of the pressure side from the stagnation downward. An increase of BR has the effect of slightly improving the effectiveness values in between the rows and on the suction side, while on the pressure side, downstream of row\#4, the highest blowing condition becomes the worst performing, also in view of the associated higher coolant consumption. This behaviour is explained by the flow separation observed in the PIV data of Fig. 2-f.

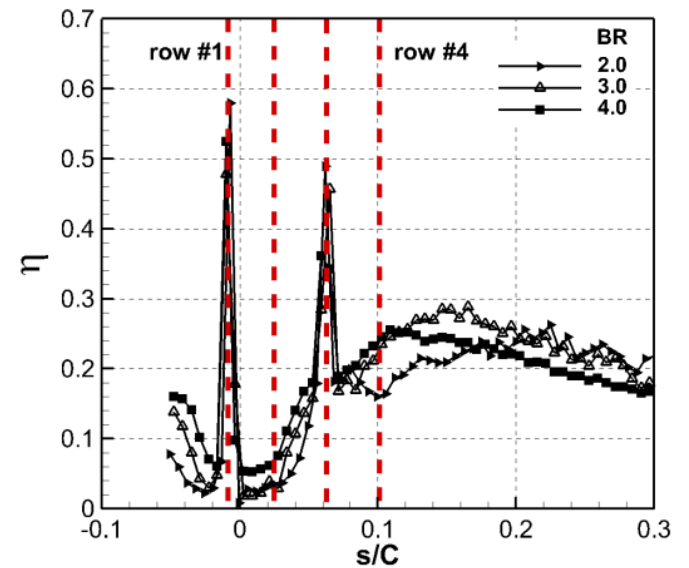

(a)

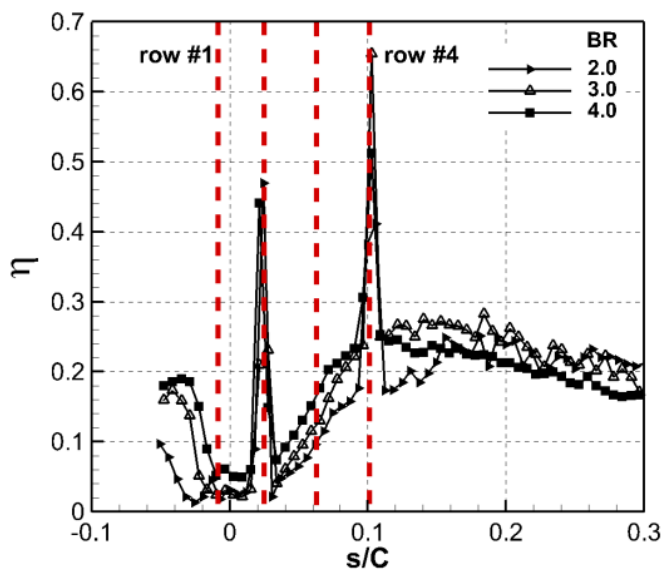

(b) and $2-4 c$ (b) for variable $B R$.

\section{Flow along the vane span}

Flow measurements have been also performed in two planes normal to the vane surface and aligned with the span wise direction, located in correspondence of row\#1 and \#4. Figure 6 reports the time averaged in plane velocity $V e l_{n z}$ distribution and the corresponding stream tracers for both planes and for all the $B R$ conditions. Due to optical accessibility constrains, the reported contour plots are limited to $60 \%$ of the vane span. In each plot, the $Z$ axis origin coincides with the vane inner hub, where coolant enters the vane internal cavity. Unavoidable problems of laser light reflection spoiled the quality of the PIV images in plane $4 \mathrm{~s}$ in the region closed to the vane surface and in the span range $\mathrm{Z} / \mathrm{H}=0.35-0.45$. In this area, low velocity and high fluctuations (see Fig. $7 b-\mathrm{d}-\mathrm{f}$ ) are measured, in particular for the case at $\mathrm{BR}=4$, and are not physical.

Starting from the case at lower $B R$, in plane 1s (Fig 6a) the stream tracers path highlights the coolant displacement towards the outer hub consequent to the selected $45^{\circ}$ injection angle. In plane velocity distribution reports a velocity deficit near the inner hub while, moving towards the outer hub, the footprints of coolant jets are well captured. The jet core is observed away from the wall low velocity region, indicating jet lift off immediately out of the injection point consistently with the observation made about the data in the blade-to-blade plane 1-3c. However, the mean path of the stream tracers suggests that after detaching from the vane surface, the jets bend towards the outer hub and reattach on the wall. This is more evident in plane 4s (Fig. 6b), where conversely the jets appear less intense and more diffused with respect to what observed in plane $1 \mathrm{~s}$. The reason of this must be ascribed to the orientation of the measurement plane with respect to the main flow direction. In plane $1 \mathrm{~s}$ the main flow is aligned along the plane (i.e is almost orthogonal to the vane surface, see Fig. 2a$\mathrm{b}-\mathrm{c})$. This does not happen in plane $4 \mathrm{~s}$ where the main flow direction is crosswise the measurement plane (Fig. 2d-e-f) and so will be the jet cores. Consequently, the jets principal velocity cannot be measured in that plane. The contour plots of the in plane velocity also show that the coolant layer extends widely into the main stream, up to about $6 D$ from the vane surface. This is a consequence of the accumulation of coolant coming from the upstream injection rows \#2 and \#3. Location of plane $4 \mathrm{~s}$ is also sufficiently downstream of the leading edge to allow the identification of the horse-shoe vortex pressure side branch, whose time averaged position and extension is depicted by the stream 
tracers. The velocity distribution in the region interested by the vortex indicates that it prevents the first holes to properly discharge the coolant. At increasing $B R$, the situation described for $B R=2$ is confirmed and some particular features can be further enhanced due to the highest coolant velocity. In particular:
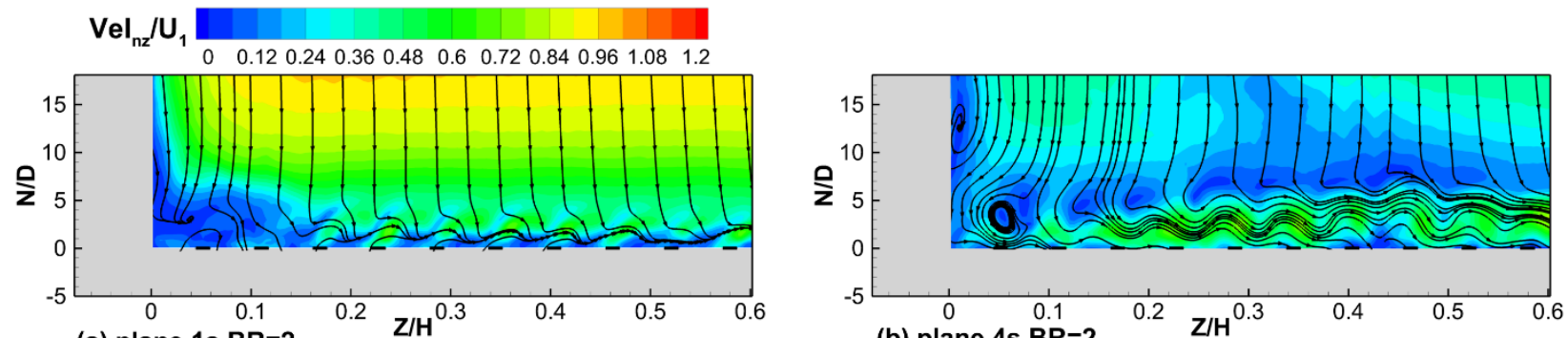

(a) plane 1s BR=2
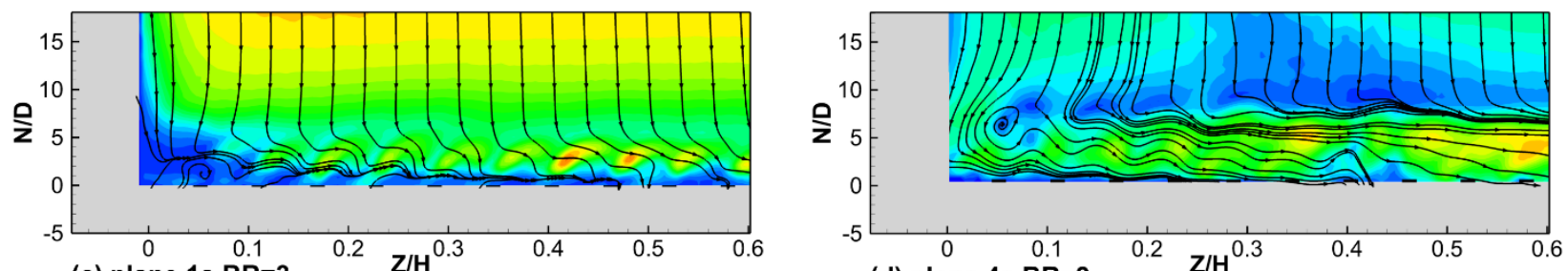

(c) plane 1s $B R=3$

(d) plane $4 \mathrm{~s} B R=3$
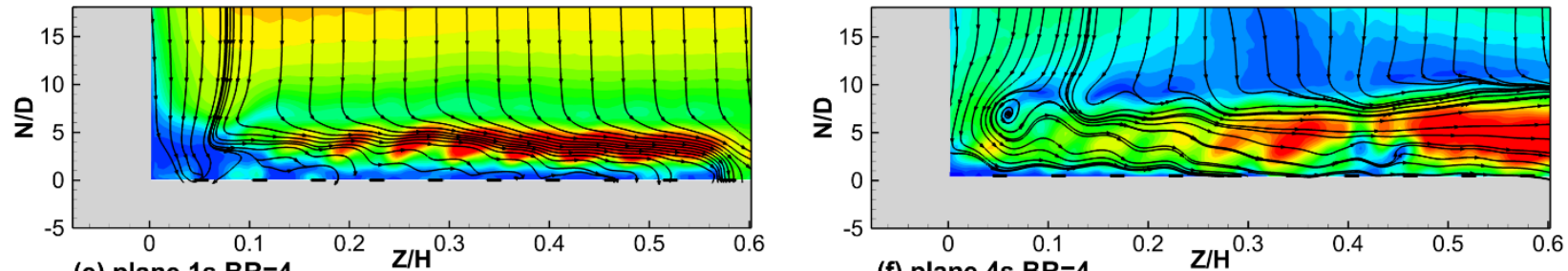

(e) plane 1s BR=4

(f) plane $4 \mathrm{~s} B R=4$

Figure 6: In plane normalized velocity distributions for variable $B R$ on the two planes 1s (ac) and $4 \mathrm{~s}(\mathrm{~d}-\mathrm{e})$.

- jet footprints become more evident and obviously characterized by higher velocity;

- in plane $4 \mathrm{~s}$ the coolant layer becomes wider, extending up to $8 D$ and $10 D$ for $B R=3$ and 4 , respectively.

- also at the $B R=3$ and 4 , the horseshoe vortex maintains a blockage effect on the first two holes of row \#4 (see velocity distribution in Fig. 6).

In plane rms velocity fluctuations were used to compute the turbulence intensity distributions reported in Fig. 7. The spike of high fluctuations that can be observed in Fig. 7a at Z/H about 0.1 and extending inside the main stream is due to bad laser light reflections. High levels of flow agitation are generally observed, consistently with blade-to-blade data (compare with Fig. 3), with increasing intensity at rising $B R$. At fixed $B R$, the highest levels are measured in plane $1 \mathrm{~s}$, but this is again the effect of the alignment between main flow velocity/measurement plane commented above when introducing the in-plane velocity distributions.

Very low levels of fluctuations, comparable to those of the incoming main flow, are measured in the region of the vane to endwall junction of plane 1s, where the suction side leg of horseshoe vortex is going to develop. In the same region of plane $4 \mathrm{~s}$ velocity fluctuations are higher than those in plane $1 \mathrm{~s}$ but however lower than those measured at higher elevation along the span. These low fluctuations confirm the poor coolant discharge from the first holes of the two rows. In plane $4 \mathrm{~s}$ and for $B R=4$, spots of high velocity fluctuations are detected at the boundaries of the coolant layer (at about $N / D=8$ ). They are the traces of jets coming from the upstream rows. This feature can be captured also for $B R=3$ while it is not observed for $B R=2$. 


\section{CONCLUSIONS}

The results of this aerodynamic investigation give a comprehensive view of jet to mainstream mixing process in the showerhead leading edge region for variable injection conditions. Different planes were investigated, providing a $3 \mathrm{D}$ view of the leading edge mean and turbulent flow field. PIV data confirmed the shift of stagnation towards the suction side for all investigated injection conditions, with only one row injecting towards the suction side. Jet lift off phenomena become relevant increasing the injection condition, coupled with increasing levels of turbulence, due to the strong jet to jet and jet to mainstream mixing process. The unsteady nature of showerhead cooling was well documented by looking at the instantaneous vorticity contours. No coherent structures could be identified, supporting the random nature of mixing process. Finally, the results presented in this paper will be useful for CFD validation.
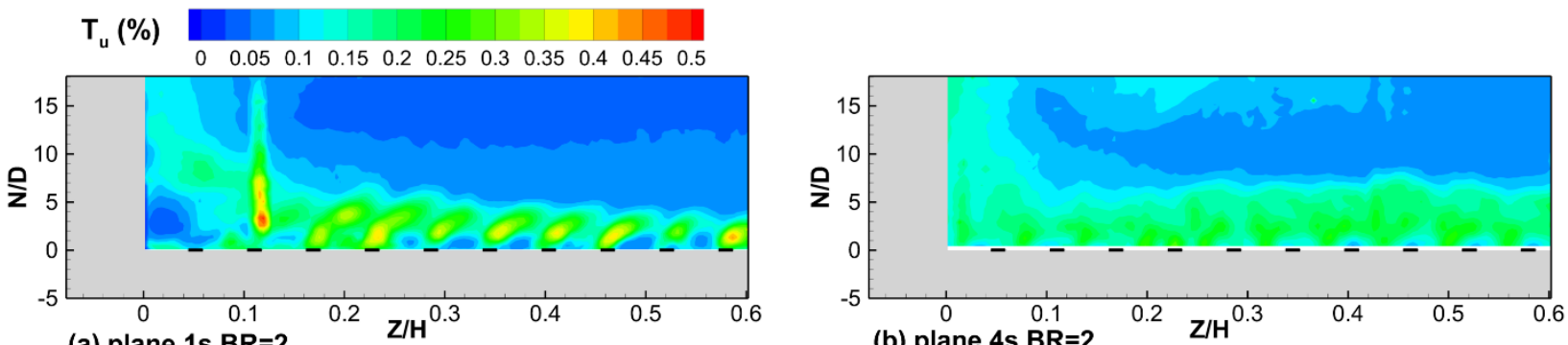

(a) plane 1s BR=2
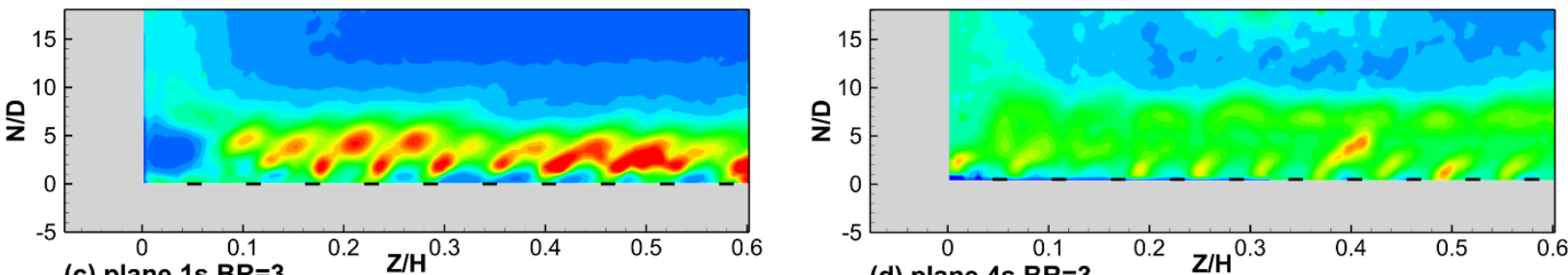

(c) plane 1s BR=3

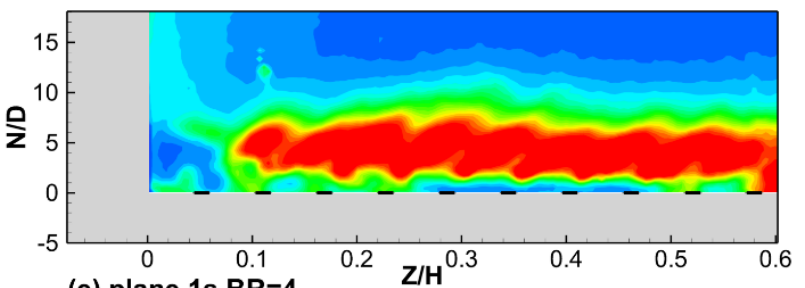

(d) plane $4 \mathrm{~s} B R=3$

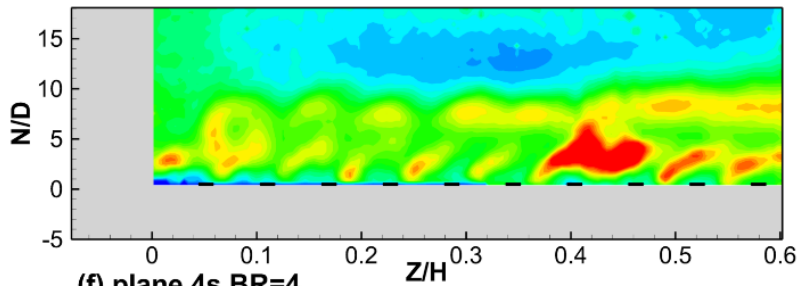

(e) plane 1s BR=4

(f) plane $4 \mathrm{~s} B R=4$

Figure 7: In plane normalized velocity distributions for variable $B R$ on the two planes 1s (ac) and $4 s(d-e)$.

\section{ACKNOWLEDGEMENTS}

The authors are indebted to Prof. A. Perdichizzi for his invaluable support.

\section{REFERENCES}

Barigozzi, G. and Ravelli, S., (2015). Combined Experimental and Numerical Study of Showerhead Film Cooling in a Linear Nozzle Vane Cascade. ASME Paper GT2015-42397.

Barigozzi G., Casarsa L., Pagnacco F., Rouina S., (2019). Experimental Investigation of the interaction between showerhead coolant jets and main flow. Experimental Thermal and Fluid Science, in press, https://doi.org/10.1016/j.expthermflusci.2019.02.014

Bogard, D.G., Thole, K.A., (2006). Gas Turbine Film Cooling. AIAA Journal of Propulsion and Power 22(2), 249-271. 
Bohn D.E., Becker V.J., Rungen A.U., (1997). Experimental and Numerical Conjugate Flow and Heat Transfer Investigation of a Shower-head Cooled Turbine Guide Vane. ASME Paper No. 97-GT15.

Chavez, K., Slavens, T.N., and Bogard, D., (2017). Experimentally Measured Effects of Incidence Angles on the Adiabatic and Overall Effectiveness of a Fully Cooled Turbine Airfoil with Shaped Showerhead Holes. J. Turbomach., 139, p 091007.

Cutbirth J.M., Bogard D.G., (2001). Thermal field and flow visualization within the stagnation region of a film cooled turbine vane. ASME paper 2001-GT-0401.

Dyson, T.E., Bogard, D.G., Bradshaw, S.D., (2012). Evaluation of CFD Simulations of Film Cooling Performance in the Showerhead Region of a Turbine Vane Including Conjugate Effects. ASME Paper No. IMECE2012-88386.

Jenkins, S.C., Varadarajan, K., Bogard, D.G., (2004). The Effects of High Mainstream Turbulence and Turbine Vane Film Cooling on the Dispersion of a Simulated Hot Streak. ASME J. Turbomach, 126, pp. 203-211.

Li, S.J., Yang, S.F., Han, J.C., (2013). Effect of Coolant Density on Leading Edge Showerhead Film Cooling Using PSP Measurement Technique. ASME Paper GT 2013-94189.

Mhetras, S., Han, J.C., Rudolph, R., (2012). Effect of Flow Parameters Variations on Full Coverage Film-cooling Effectiveness for a Gas Turbine Blade. J. Turbomach., 134(1), p.011004.

Polanka M.D., Cutbirth J.M., Bogard D.G., (2002). Three component velocity field measurements in the stagnation region of a film cooled turbine vane. J. Turbomach., 124, pp. 445-452.

Ravelli S., Barigozzi G., 2017, Comparison of RANS and Detached Eddy Simulation Modeling Against Measurements of Leading Edge Film Cooling on a First-Stage Vane. J. Turbomach., 139, 051005.

Rozati, A. and Tafti, D.K., (2008). Large-Eddy Simulations of Leading Edge Film Cooling: Analysis of Flow Structures, Effectiveness and Heat Transfer. Int. J. Heat Fluid Flow, 29(1), pp. 117. 